\title{
Distribución estomática en maíz a diferentes dosis de humus
}

\section{Statomatic distribution in corn to different dose of humus}

\author{
CASARRUBIAS-CASTILLO, Kena†*, LOZA-LLAMAS, Juana América, ZAÑUDO-HERNÁNDEZ, \\ Julia y MÉNDEZ-MORÁN, Lucila.
}

Centro Universitario de Ciencias Biológicas y Agropecuarias, Universidad de Guadalajara

ID $1^{\text {er }}$ Autor: Kena, Casarrubias-Castillo/ ORC ID: 0000-0003-1831-8642, Researcher ID Thomson: G-6739-2018, CVU CONACYT ID: 227935

ID $1^{\text {er }}$ Coautor: Juana A., Loza-Llamas / ORC ID: 0000-0003-1135-0754, Researcher ID Thomson: U-1402-2018, CVU CONACYT ID: 924239

ID $2^{\text {do }}$ Coautor: Julia, Zañudo-Hernández / ORC ID: 0000-0002-0834-6626, CVU CONACYT ID: 201106

ID $3^{\text {er }}$ Coautor: Lucila, Méndez-Morán / ORC ID: 0000-0003-4733-6153, Researcher ID Thomson: U-1401-2018, CVU CONACYT ID: 121862

DOI: $10.35429 / J O T I .2019 .11 .3 .23 .26$

Recibido 16 de Julio, 2019; Aceptado 19 de Septiembre, 2019

\section{Resumen}

El maíz es el cultivo de mayor importancia en México, el cual es el centro de origen y diversidad, además de formar parte importante de su alimentación y cultura presentando una profunda relación con su economía. Las proyecciones climatológicas y de erosión del suelo obligan a estudiar el comportamiento del cultivo ante posibles cambios, hoy en día es importante la modificación de las prácticas agroecológicas, una de ellas es el uso de biofertilizantes orgánicos. Estudios recientes sugieren que la regulación del desarrollo de los estomas en las gramíneas podría ser utilizada para mejorar la eficacia de la planta y el rendimiento agrícola. El objetivo del presente trabajo fue evaluar el efecto del uso de abonos orgánicos a diferentes dosis de humus en la distribución estomática en plantas de maíz. En este trabajo se evaluó el número de estomas en la superficie adaxial y abaxial de las hojas, así como la longitud de los estomas de plantas de maíz crecidas con dosis de 10, 20 y 30 ton/ha de humus.

Humus, Maíz, Biofertilizante

\begin{abstract}
Resumen
Corn is the most important crop in Mexico, is the center of origin and diversity, as well as being an important part of its diet and culture, representing a deep relationship with its economy.Climatic and soil erosion projections force the study of the behavior of the crop in the face of possible changes, nowadays the modification of agroecological practices is important, one of them is the use of organic biofertilizers. Recent studies that determine the regulation of stomata development in grasses could be determined to improve plant efficiency and agricultural yield. The objective of the present work was to evaluate the effect of the use of organic fertilizers at different doses of humus in the stomatic distribution in corn plants. In this work the number of stomata in both the abaxial and the adaxial of the leaves was evaluated, as well as the length of stomata of corn plants grown with doses of 10,20 and 30 ton/ha of humus.
\end{abstract}

Humus, Corn, Biofertilizer

Citación: CASARRUBIAS-CASTILLO, Kena, LOZA-LLAMAS, Juana América, ZAÑUDO-HERNÁNDEZ, Julia y MÉNDEZ-MORÁN, Lucila. Distribución estomática en maíz a diferentes dosis de humus. Revista de Invención Técnica 2019. 3-11:23-26

\footnotetext{
*Correspondencia al Autor (Correo Electrónico: kena.casarrubias@ academicos.udg.mx)

$\dagger$ Investigador contribuyendo como primer autor.
} 


\section{Introducción}

El maíz (Zea mays L.) es una planta perteneciente a la familia de las poáceas (Poaceae) o gramíneas, originaria y domesticada por los pueblos indígenas en el centro de México (Acosta I.2 009), siendo la base de la alimentación en México y América, junto con el frijol, calabaza y chile.

El maíz necesita para su desarrollo ciertas cantidades de elementos minerales como Potasio $(\mathrm{K})$ y Fósforo $(\mathrm{P})$ y nitrógeno $(\mathrm{N})$ y por lo general los primeros estadios de desarrollo del maíz son muy sensibles a la falta de agua y nutrientes. Por otra parte, los suelos donde se cultiva por lo general no tienen la capacidad para proporcionar los nutrientes necesarios para el crecimiento eficiente de las plantas, por cual el desarrollo se ve comprometido y no llegan a un rendimiento adecuado, para ello se debe recurrir al empleo de fertilizantes químicos o inorgánicos (OECD 2003; Mariscal-Nava 2019).

La lombricultura es un método para generar materia orgánica, y que permite combatir la degradación de los suelos y aumentar la producción de los cultivos sin contaminar el medioambiente.

Se ha evaluado el efecto de la aplicación de humus de lombriz sobre la productividad del cultivo y se han observado cambios en las características del suelo (Méndez-Moreno et al., 2012; Flores-Chávez 2014).

La familia a la que pertenece el maíz, posee estomas que tienen forma de mancuerna y están alineados en filas a lo largo de la lámina de la hoja de la planta, estudios siguieren que la regulación del desarrollo de los estomas en las gramíneas podría ser utilizada para mejorar la eficacia de la planta y el rendimiento agrícola (Raissig et al., 2016).

En este trabajo se propone la aplicación de humus en suelos a diferentes dosis y observar el efecto sobre la distribución y longitud de los estomas de plantas de maíz.

\section{Materiales y Métodos}

Material vegetal: Se utilizaron semillas de maíz Asgrow 7573.
Sustrato utilizado. El sustrato utilizado consiste en un suelo Franco-Arcillo-Arenoso, con una mezcla de humus de lombriz a razón de ton/ha sin aplicación adicional de insumos agrícolas (fertilizantes o pesticidas) ya sea al sustrato o al cultivo.

Establecimiento del experimento y tratamientos. Los experimentos se llevaron a cabo en condiciones de invernadero en el módulo de biorreciclados y sistema de producción del CUCBA. Se establecieron tres experimento para evaluar la efectividad biológica del humus de lombriz: Se utilizó un diseño experimental en bloques completos al azar con cuatro tratamientos: Tratamiento 1: 0 ton/ha de humus, Tratamiento 2: 10 ton/ha de humus, Tratamiento 3: 20 ton/ha de humus y Tratamiento 4: 30 ton/ha de humus.

Observación de estomas: Se utilizó un microscopio marca OPTIKA ITALY, las observaciones se realizaron con el objetivo 10X. Se contó el número de estomas de las superficies adaxial y abaxial de las hojas de maíz. Las fotografías se tomaron con una cámara (Optika visión life) y a partir de ellas se midió la longitud de los estomas en cada uno de los tratamientos. Estadística: Se utilizó la prueba de ShapiroWilk" y la prueba Kruskal-Wallis a $(P<0.05)$ se aplicó utilizando el Programa $\mathrm{R}$ versión 3.5.

\section{Resultados y Discusión}

Numero de estomas no cambia respecto a los tratamientos

El maíz se caracteriza por poseer hojas anfistomatosas, es decir, tiene estomas en ambos lados de la hoja, ocupan entre $0.5 \%$ y $5 \%$ de la epidermis de la hoja y son más abundantes en la superficie abaxial (cita). Se observa en la figura 1 que el número de estomas es mayor en la superficie abaxial que adaxial por la naturaleza de las hojas de maíz, sin embargo, no se observan diferencias significativas en los distintos tratamientos. 

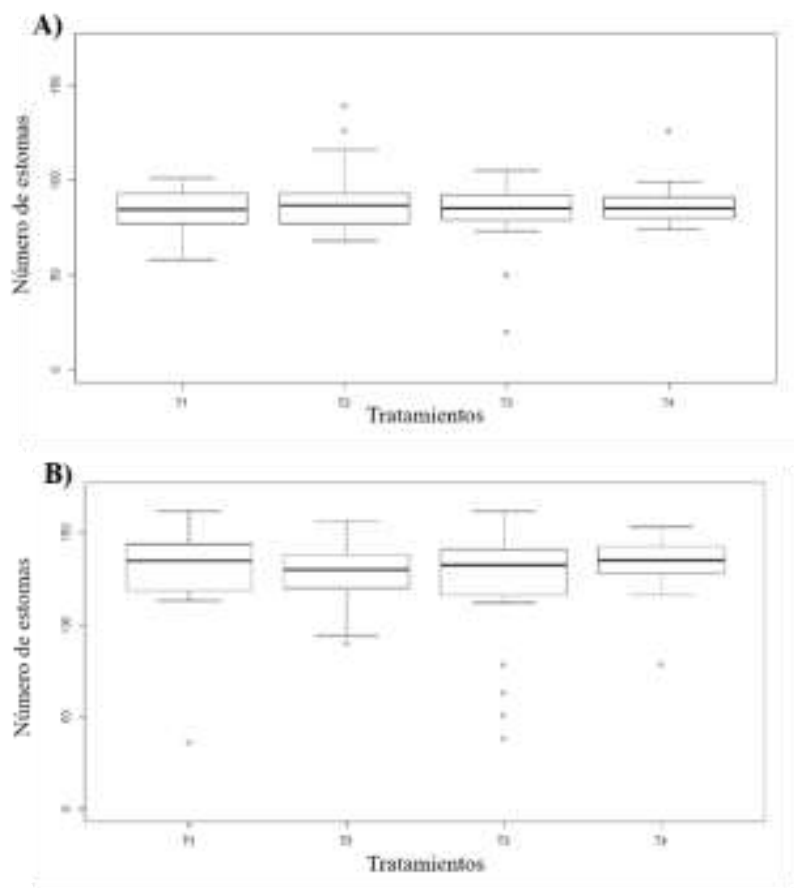

Figura 1 Número de estomas a diferentes dosis de humus. A) Superficie adaxial B) superficie abaxial.

\section{Longitud estomática a distintas dosis de} humus.

Los estomas son un tipo de células que forman una estructura tipo poro en la epidermis de las hojas, su principal función es el intercambio de gases con el medioambiente. En la figura 2 se presenta la longitud estomática de ambas superficies de las hojas de maíz, se observa que la longitud tiene una tendencia a incrementar en el tratamiento 4, sin embargo, el análisis estadístico indica que no hay diferencias entre los tratamientos.

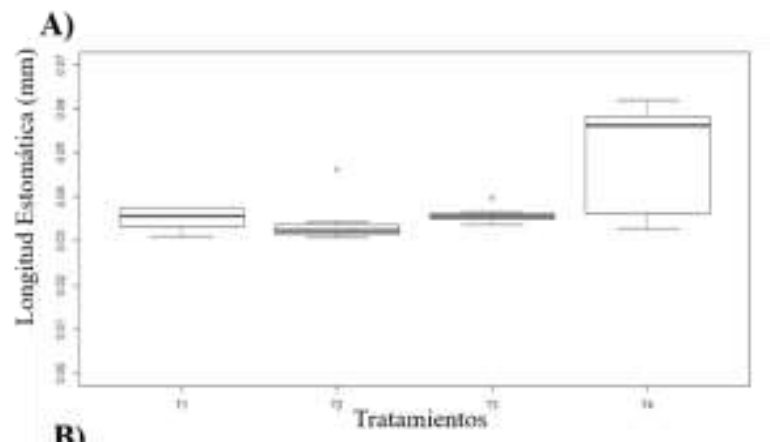

B)

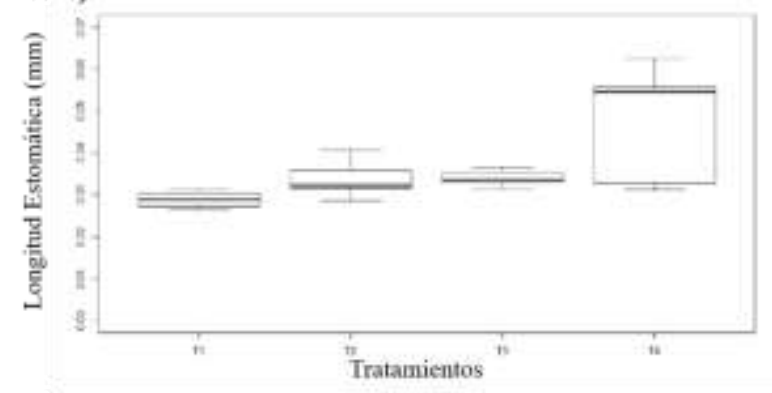

Figura 2 Longitud de estomas a diferentes dosis de humus. A) Superficie adaxial B) superficie abaxial.
Interesantemente se observó que la distribución de los estomas cambia a distintas dosis de humus, en la figura 3 se observa en el tratamiento 1 una distribución lineal y en el tratamiento 3 una distribución en zigzag de los estomas en algunas zonas.

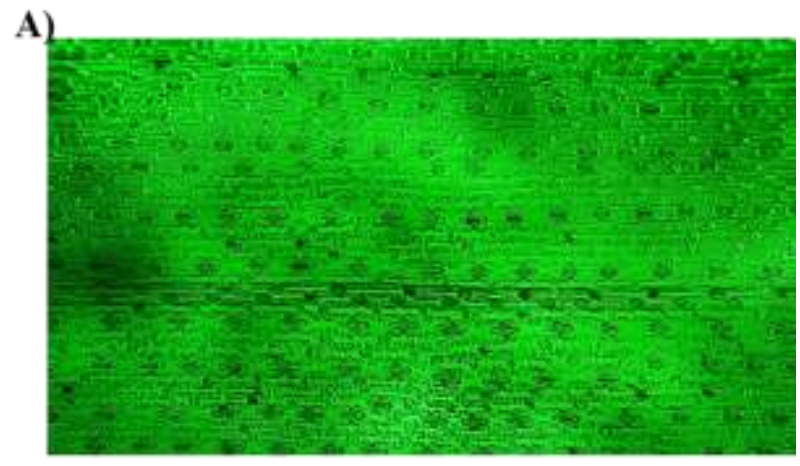

B)

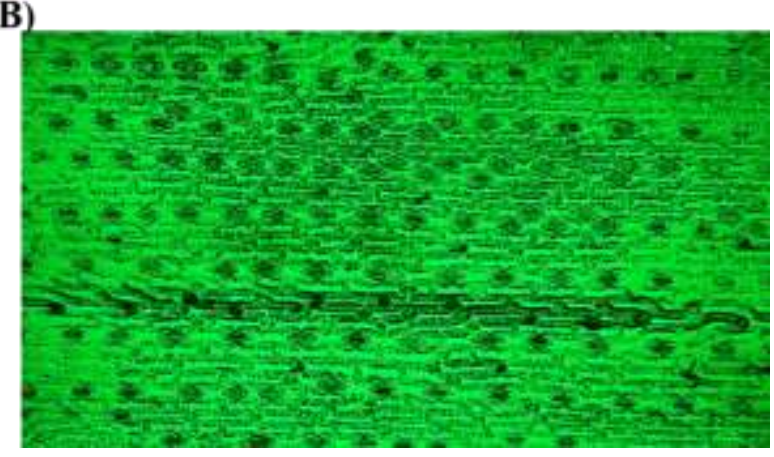

Figura 3 Distribución de estomas de hojas de maíz a diferentes dosis de humus. A) Tratamiento de 0 ton/h, B) Tratamiento de 20 ton/h.

\section{Conclusiones}

Los estomas se encuentran en la interfaz plantaatmósfera, regulan la fotosíntesis y la eficiencia del uso del agua, lo cual se traduce a que estas células pueden ser factores determinantes para aumentar la productividad de las plantas.

La prueba estadística indica que no hay diferencias en el número de estomas en las superficies abaxial y adaxial a distintas dosis de humus. Se observa una tendencia a aumentar la longitud de estomas en el tratamiento 4 con respecto al tratamiento 1 , sin embargo, no hay diferencias mínimas significativas. Este trabajo deja el panorama abierto a investigar con detenimiento, ampliar el muestreo y analizar si la aplicación de humus en suelos contribuye a cambiar las características de los estomas. 


\section{Referencias}

Acosta, Rosa. (2009). El cultivo del maíz, SU origen y clasificación. EL MAIZ en Cuba. Cultivos Tropicales, 30(2), 00. Recuperado en 18 de septiembre de 2019, de http://scielo.sld.cu/scielo.php?script=sci_arttext $\& \mathrm{pid}=\mathrm{S} 0258$ -

$59362009000200016 \& \operatorname{lng}=$ es\&tlng=es.

Flores-Chavez, Anabel. (2012). Efectividad biologica del humus de lombriz en el cultivo de Maíz y Chile en el estado de Aguascalientes. (Tesis de maestría). Universidad de Aguascalientes, México.

Mariscal-Nava Carlos R. (2019). Caracterización bioquímica de una variedad de maíz sometida a estrés abiótico. (Tesis pregrado) Universidad de Guadalajara, México.

Méndez-Moreno, O., Samuel León-Martínez, N., Antonio Gutiérrez-Miceli, F., RincónRosales, R., José, \&, \& Alvarez-Solís, D. (n.d.). Efecto de la aplicación de humus de lombriz en el crecimiento y rendimiento de grano del cultivo de maíz Effect of earthworm humus application in the maize growth and grain yield. OECD (2003). Consensus document on the biology of Zea mays subsp. mays (Maize). Report No. 27, Environment Directorate; Organisation for Economic Co-operation and Development Paris, France.

Raissig, M. T., Abrash, E., Bettadapur, A., Vogel, J. P., \& Bergmann, D. C. (2016). Grasses use an alternatively wired bHLH transcription factor network to establish stomatal identity. Proceedings of the National Academy of Sciences of the United States of America, 113(29), 8326-8331. http://doi.org/10.1073/pnas.1606728113 